\title{
Studies of the Control of Plasma Aldosterone Concentration in Normal Man
}

\author{
I. RESPONSE TO POSTURE, ACUTE AND CHRONIC \\ VOLUME DEPLETION, AND SODIUM LOADING
}

\author{
Gordon H. Williams, John P. Cain, Robert G. Dluhy, and \\ RICHARD H. UNDERWOOD \\ From the Endocrine-Metabolic Unit, Peter Bent Brigham Hospital, \\ and the Department of Medicine, Harvard Medical School, \\ Boston, Massachusetts 02115
}

A в STRACT The peripheral plasma levels of aldosterone, renin activity (PRA), potassium, corticosterone, cortisol, and in some cases angiotensin II, were measured in normal subjects undergoing postural changes, acute diuretic-induced volume depletion, and alterations in dietary sodium. On a $10 \mathrm{mEq}$ sodium $/ 100 \mathrm{mEq}$ potassium intake, subjects supine for 3 consecutive days had identical diurnal patterns of PRA, angiotensin II, aldosterone, cortisol, and corticosterone, with peaks at $8 \mathrm{a} . \mathrm{m}$. and nadirs at $11 \mathrm{p} . \mathrm{m}$. With an increase in sodium intake to $200 \mathrm{mEq}$, plasma levels of aldosterone and PRA fell to one-third their previous levels but the diurnal pattern in supine subjects was unchanged and again parallel to that of cortisol and corticosterone. There was no diurnal variation of plasma potassium on either sodium intake in the supine subjects. On a $10 \mathrm{mEq}$ sodium $/ 100 \mathrm{mEq}$ potassium intake, supine 8 a.m. plasma aldosterone $(55 \pm 7 \mathrm{ng} / 100 \mathrm{ml})$ and PRA $(886 \pm 121 \mathrm{ng} /$ $100 \mathrm{ml}$ per $3 \mathrm{hr}$ ) increased by $150-200 \%$ after subjects were upright for $3 \mathrm{hr}$. However, even though the patients maintained an upright activity pattern, there was a significant fall in plasma aldosterone to $33 \pm 5 \mathrm{ng} / 100 \mathrm{ml}$ at 11 p.m. Potassium levels varied in a fashion parallel to aldosterone and PRA. Plasma cortisol and corticosterone had a diurnal pattern similar to that found in supine subjects. In response to acute diuretic-induced volume de-

This work was presented in part at the 53rd Annual Meeting of The Endocrine Society, 25 June 1971, San Francisco, Calif.

Dr. Williams is an Investigator of the Howard Hughes Medical Institute.

Received for publication 3 December 1971 and in revised form 18 February 1972. pletion, the nocturnal fall in aldosterone levels did not occur. The 11 p.m. value $(102 \pm 20 \mathrm{ng} / 100 \mathrm{ml})$ and the 8 a.m. value postdiuresis $(86 \pm 15 \mathrm{ng} / 100 \mathrm{ml})$ were both significantly greater than the prediuresis levels. PRA showed a similar altered pattern while potassium levels fell throughout the day. In some but not all studies, changes in plasma aldosterone coincided with changes in plasma cortisol, corticosterone, and/or potassium. However, in all studies, changes in plasma aldosterone were invariably associated with parallel changes in plasma renin activity and/or angiotensin II levels. These findings support the concept that PRA is the dominant factor in the control of aldosterone when volume and/or dietary sodium is altered in normal man.

\section{INTRODUCTION}

Alterations of aldosterone secretion in response to sodium restriction, posture, acute diuresis, or hemorrhage have been widely considered to be mediated by the reninangiotensin system (1-4). Other factors, such as potassium (5-7), adrenocorticotropin (ACTH) (7-9), and serotonin (10) have been shown to alter aldosterone secretion in vivo and/or in vitro, but presumably, they play only a minor role in comparison to the renin-angiotensin system in controlling the secretion of aldosterone in response to volume changes. However, some investigators (11-13) have suggested that the renin-angiotensin system is not necessarily the primary mechanism leading to increased secretion of aldosterone during sodium or volume changes. Disagreement on this point could be related to the usual method of studying these two parameters. Most previous studies in man have related either 
urinary excretion or secretion rates of aldosterone determined over hours to single or multiple but instantaneous determinations of renin activity or angiotensin II levels $(3,4,6,8,14)$. The exact relationship of renin activity to plasma aldosterone when changes in volume are produced have not been entirely clear from the few previous studies when both have been measured simultaneously $(13,15,16)$ and none have measured both potassium and ACTH levels, directly or indirectly, at the same time. In order to clarify this relationship the present paper reports the simultaneous measurement of plasma corticosterone, cortisol, aldosterone, potassium, angiotensin II, and peripheral renin activity (PRA) ${ }^{1}$ in a group of normal subjects undergoing postural changes, acute diuretic-induced volume depletion and alterations in dietary sodium.

\section{METHODS}

\section{Protocols}

13 normal subjects ( 11 males, 2 females) between the ages of 21 and $30 \mathrm{yr}$ were studied on The Clinical Research Center of the Peter Bent Brigham Hospital. All subjects were normotensive, had normal physical examinations, and no evidence by history or laboratory testing for clinical disease. All denied the use of drugs. All were informed of the nature of the studies and signed consent forms were obtained. Each subject underwent one or more of the following protocols.

\section{Constant Activity-Constant Diet}

Chronic (low dietary sodium) volume-depletion study (10 $m E q N a, 100 m E q K$ diet). Five subjects (four males, one female) were maintained on a constant activity pattern simulating normal daily activity, i.e., supine from 11:30 p.m. until 8 a.m.; up from 8 a.m. to $11: 30$ p.m. with one 300 yard walk at least hourly. All subjects ate a constant 10 $\mathrm{mEq}$ sodium $/ 100 \mathrm{mEq}$ potassium, $2500 \mathrm{ml}$, isocaloric diet divided into three meals a day and a small snack at bedtime. On the day of admission, $400-500 \mathrm{ml}$ of blood was drawn from each subject in a sterile acid citrate dextrose blood bank container and maintained in the blood bank for reinfusion later in the study.

After they had achieved metabolic balance (usually on the 5th or 6th day), blood was obtained for sodium, potassium, hematocrit, total protein, plasma cortisol, corticosterone, aldosterone, and PRA at 8 a.m., 11 a.m., 5 p.m., 11 p.m., and at 8 a.m. the following morning. PRA was not determined at 11 p.m. After each sample of blood was drawn, an equivalent volume (approximately $60-80 \mathrm{ml}$ ) of the patient's blood was reinfused. All blood samples were drawn premeal or presnack. Urines were collected every 6 $\mathrm{hr}$ and analyzed for creatinine, sodium, and potassium.

Acute diuretic-induced volume-depletion study (10 $\mathrm{mEq}$ $N a, 100 \mathrm{mEq} K$ diet). This study began the day after the chronic volume depletion-constant activity protocol with the same five subjects maintained on the same dietary intake and activity program. Blood was drawn and replaced and urine collected for the same determinations in an identical

\footnotetext{
${ }^{1}$ Abbreviation used in this paper: PRA, plasma renin activity.
}

fashion as in the previous study. At 9 a.m., the subjects were given oral furosemide every $6 \mathrm{hr}$ (mean total dose $=190 \mathrm{mg} / 24 \mathrm{hr}$ ) to maintain a sufficient urine output to produce a $1.5-2 \mathrm{~kg}$ weight loss over the $24 \mathrm{hr}$ period (17).

\section{Supine Study-Constant Diet}

Chronic (low dietary sodium) volume-depletion study (10 $m E q N a, 100 m E q K$ diet). On the day of admission 700$800 \mathrm{ml}$ of blood was drawn from five male subjects and stored in the blood bank for reinfusion later in the study. When the subjects had achieved metabolic balance on the $10 \mathrm{mEq}$ sodium, $100 \mathrm{mEq}$ potassium diet, they were placed in the supine position for 82 consecutive $\mathrm{hr}$, beginning at 10 p.m. the day before sampling. Daily blood samples for plasma potassium, corticosterone, cortisol, aldosterone, and PRA and in some cases angiotensin II levels were drawn at 8 a.m., 11 a.m., 5 p.m., and 11 p.m. on 3 consecutive days. After each sampling an equivalent amount of blood was replaced.

Volume expansion study (200 mEq Na/200 mEq K diet). Five subjects (four males, one female) were phlebotomized upon admission. When they achieved metabolic balance on the $200 \mathrm{mEq}$ sodium $/ 200 \mathrm{mEq}$ potassium diet, they were placed in the supine position for 82 consecutive hr. Blood samples were drawn at 8 a.m., 11 a.m., 5 p.m., and 11 p.m. for plasma cortisol, corticosterone, aldosterone, potassium, and PRA. Equivalent amounts of blood were reinfused as described in the previous protocol.

\section{Laboratory procedures}

All blood samples were immediately spun and the plasma separated and frozen until time for assay. The samples for PRA and angiotensin II levels were drawn using ethylenediaminetetraacetic acid (EDTA) as the anticoagulant and the samples for potassium, corticosterone, cortisol, and aldosterone used sodium heparin as the anticoagulant. Sodium and potassium in urine and blood were measured by flame photometry using lithium as an internal standard. Plasma cortisol and corticosterone levels in the two constant activity studies were measured by a modification of the doubleisotope derivative technique of $\mathrm{Kliman}$ and Peterson (18). In this method the nonspecific blank was $200 \mathrm{pg} / 2 \mathrm{ml}$, the coefficient of variation at the 20 and $2 \mathrm{ng} / \mathrm{ml}$ level, respectively was $15-20 \%$, and the recovery of added steroid ranged from 75 to $105 \%$. The plasma aldosterone levels in the two constant activity studies were measured by a double-isotope derivative technique as described by Brodie, Shimizu, Tait, and Tait (19). The nonspecific blank for this method in our laboratory is $105 \pm 85$ ( $\mathrm{SD}$ ) $\mathrm{pg} / 20 \mathrm{ml}$ with a coefficient of variation at the $5 \mathrm{ng} / 100 \mathrm{ml}$ level of $\pm 15 \%$.

The plasma aldosterone, corticosterone, and cortisol in the two supine studies were measured by displacement analysis techniques as previously described from this laboratory (20). In brief, the plasma is extracted with methylene chloride, chromatographed in a Bush 5 system and the three steroids eluted. The corticosterone and aldosterone are determined by radioimmunoassay using antibodies specifically directed against them. The cortisol is measured by a competitive protein-binding method similar to that described by Nugent and Mayes (21). The levels found in the plasma of adrenalectomized subjects are below the sensitivity of the method for cortisol and corticosterone, and at the $2 \mathrm{pg} / 4 \mathrm{ml}$ level for aldosterone. The sensitivity of the cortisol assay system is $0.2 \mathrm{ng} /$ binding tube; for the corticosterone assay, $0.02 \mathrm{ng} / \mathrm{binding}$ tube; and for the aldosterone, $0.002 \mathrm{ng} /$ binding tube. The coefficient of variation is between 7 and 
$11 \%$ at a level 10 times the sensitivity for the three assays. Recovery of added steroid from each assay is the same ranging from 85 to $110 \%$. The cortisol, corticosterone, and aldosterone values in 12 samples were compared with the above mentioned double-isotope derivative method which produced a correlation coefficient of 0.99 with a $P$ value of correlation $<0.001$ for all three steroids.

Angiotensin II values were measured by a double-antibody radioimmunoassay system (22). This assay is sensitive to a level of $10 \mathrm{pg} / \mathrm{ml}$ with a coefficient of variation at the 20 $\mathrm{pg} / \mathrm{ml}$ level of $6.8 \%$. Recoveries of added angiotensin II at three different levels range from 88 to $108 \%$.

PRA was measured by a modification of the Boucher method as previously described (17). Statistical analyses were performed as described by Snedecor and Cochran (23), utilizing a General Electric 635 computer. The results are expressed as mean \pm standard error of the mean and significance as $P<0.02$ unless otherwise indicated. Nonsignificant differences were those with $P>0.05$.

\section{RESULTS}

\section{Constant activity}

Chronic (low dietary sodium) volume depletion. Table I summarizes the results from this study. The mean $24 \mathrm{hr}$ urinary sodium excretion was $6 \pm 1.4 \mathrm{mEq} / 24 \mathrm{hr}$ and potassium excretion, $94 \pm 4 \mathrm{mEq} / 24 \mathrm{hr}$. There was no significant variation in the $6 \mathrm{hr}$ sodium excretion (range $0.6-2.4 \mathrm{mEq}$ ). However, the 7 a.m. to 1 p.m. and 1 to 7 p.m. potassium excretion $(28 \pm 2 \mathrm{mEq}$ each) was significantly greater than the 1 to 7 a.m. excretion $(14 \pm 4 \mathrm{mEq})$.

The mean 8 a.m. supine plasma aldosterone level $(55 \pm 7 \mathrm{ng} / 100 \mathrm{ml})$ rose significantly at 11 a.m. (100 \pm 17 $\mathrm{ng} / 100 \mathrm{ml}$ ) after the subjects had been upright for $3 \mathrm{hr}$. However, even though the patients maintained an upright activity pattern from 11 a.m. to 11 p.m., there was a significant fall to a 11 p.m. value of $33 \pm 5 \mathrm{ng} / 100 \mathrm{ml}$. The 11 p.m. value was also significantly less than both the 5 p.m. upright level $(68 \pm 4 \mathrm{ng} / 100 \mathrm{ml})$ and the $8 \mathrm{a} . \mathrm{m}$. supine value.
The mean plasma cortisol value showed the expected diurnal fall during the day. The $8 \mathrm{a} . \mathrm{m}$. value $(12.3 \pm 1.1$ $\mu \mathrm{g} / 100 \mathrm{ml}$ ) was significantly greater than the 11 p.m. value $(4.1 \pm 1.3 \mu \mathrm{g} / 100 \mathrm{ml})$. The plasma corticosterone values showed a similar fall with the 8 a.m. level (0.66士 $0.09 \mu \mathrm{g} / 100 \mathrm{ml}$ ) being significantly greater than the 11 p.m. value $(0.9 \pm 0.04 \mu \mathrm{g} / 100 \mathrm{ml})$.

The mean 8 a.m. supine PRA $(886 \pm 121 \mathrm{ng} / 100 \mathrm{ml}$ per $3 \mathrm{hr})$ was significantly less $(P<0.05)$ than the 11 a.m. value $(1200 \pm 185 \mathrm{ng} / 100 \mathrm{ml}$ per $3 \mathrm{hr})$ when the subjects had been upright for $3 \mathrm{hr}$. However, there was no significant difference between the 5 p.m. value (1348 \pm $226 \mathrm{ng} / 100 \mathrm{ml}$ per $3 \mathrm{hr}$ ) and either the $8 \mathrm{a} . \mathrm{m}$. or the 11 a.m. levels even though the mean level was greater. The greater standard error caused by some subjects showing a fall and others a rise from 11 a.m. to 5 p.m. is the reason for this. PRA was not determined at 11 p.m.

Potassium changed during the day in a pattern similar to the plasma aldosterone with a rise at 11 a.m. and then a gradual fall throughout the rest of the day. The 11 a.m. value $(4.8 \pm 0.1 \mathrm{mEq} /$ liter $)$ was significantly greater $(P<0.05)$ than the $11 \mathrm{p} . \mathrm{m}$. value $(4.4 \pm 0.2 \mathrm{mEq} /$ liter $)$. No significant changes in serum sodium, protein, or hematocrit occurred during the study.

Acute diuretic-induced volume depletion. The mean weight loss produced by the diuretic was $1.8 \pm 0.1 \mathrm{~kg}$ over the $24 \mathrm{hr}$ period. There was an increase in the mean sodium excretion to $202 \pm 11 \mathrm{mEq} / 24 \mathrm{hr}$ and the mean potassium excretion to $152 \pm 6 \mathrm{mEq} / 24 \mathrm{hr}$. In all 6-hr time periods there was a significantly greater sodium excretion on the diuretic day than on the control day. Also, there was a significantly greater sodium and potassium excretion during the first $12 \mathrm{hr}$ compared with the second $12 \mathrm{hr}$ on the diuretic day. This is probably explained by the fact that most of the total furosemide was given during the first $12 \mathrm{hr}$ period.

As in the control study, there was a significant in-

TABLE I

Diurnal Plasma Cortisol, Corticosterone, Aldosterone and Renin Activity, and Serum Potassium in Normal Subjects on a $10 \mathrm{mEq} \mathrm{Na/100} \mathrm{mEq} \mathrm{K} \mathrm{Intake} \mathrm{with} \mathrm{Normal} \mathrm{Activity}$

\begin{tabular}{|c|c|c|c|c|c|c|c|c|c|c|c|c|c|c|c|c|c|c|c|}
\hline \multirow{2}{*}{$\begin{array}{l}\text { Sub- } \\
\text { jects }\end{array}$} & \multicolumn{4}{|c|}{ Cortisol } & \multicolumn{4}{|c|}{ Corticosterone } & \multicolumn{4}{|c|}{ Aldosterone } & \multicolumn{4}{|c|}{ Potassium } & \multicolumn{3}{|c|}{ Renin activity } \\
\hline & $8 a^{*}$ & $11 \mathrm{a}$ & $5 p$ & $11 p$ & $8 a^{*}$ & $11 \mathrm{a}$ & $5 p$ & $11 p$ & $8 \mathrm{a} *$ & $11 \mathrm{a}$ & $5 p$ & $11 p$ & $8 a *$ & $11 \mathrm{a}$ & $5 p$ & $11 p$ & $8 a^{*}$ & $11 \mathrm{a}$ & $5 p$ \\
\hline & \multicolumn{4}{|c|}{$\mu \mathrm{g} / 100 \mathrm{ml}$} & \multicolumn{4}{|c|}{$\mu \mathrm{g} / 100 \mathrm{ml}$} & \multicolumn{4}{|c|}{$\mathrm{ng} / 100 \mathrm{ml}$} & \multicolumn{4}{|c|}{$m E q /$ liter } & \multicolumn{3}{|c|}{$\mathrm{ng} / 100 \mathrm{ml}$ per $3 \mathrm{hr}$} \\
\hline 1 & 11.7 & 7.1 & 4.0 & 1.4 & 0.38 & 0.31 & 0.22 & 0.09 & 70 & 165 & 87 & 39 & 4.5 & 4.8 & 4.4 & 4.2 & 784 & 1350 & 1150 \\
\hline 2 & 8.2 & 4.9 & 2.9 & 1.1 & 0.64 & 0.49 & 0.46 & 0.19 & 33 & 52 & 16 & 14 & 4.2 & 4.6 & 4.6 & 4.1 & 1140 & 1200 & 1000 \\
\hline 3 & 14.1 & 一 & 6.6 & 3.5 & 0.85 & - & 0.45 & 0.32 & 42 & 94 & 52 & 36 & 5.1 & 5.0 & 4.6 & 4.2 & 1210 & 1730 & 770 \\
\hline 4 & 14.9 & 7.7 & - & 6.0 & 0.92 & 0.22 & 一 & - & 80 & 109 & 84 & 34 & 4.7 & 4.8 & 4.7 & 5.0 & 975 & 1100 & 1920 \\
\hline 5 & 12.6 & 10.2 & 8.8 & 8.4 & 0.49 & 0.22 & 0.15 & 0.17 & 46 & 78 & 100 & 44 & 4.6 & 4.7 & 4.8 & 4.4 & 325 & 620 & 1900 \\
\hline Mean & 12.3 & 7.5 & 5.6 & 4.1 & 0.66 & 0.29 & 0.32 & 0.19 & 55 & 100 & 68 & 33 & 4.6 & 4.8 & 4.6 & 4.4 & 886 & 1200 & 1348 \\
\hline \pm SEM & \pm 1.1 & \pm 0.9 & \pm 1.1 & \pm 1.3 & \pm 0.09 & \pm 0.06 & \pm 0.07 & \pm 0.04 & \pm 7 & \pm 17 & \pm 14 & \pm 5 & \pm 0.1 & \pm 0.1 & \pm 0.1 & \pm 0.2 & \pm 121 & \pm 185 & \pm 226 \\
\hline
\end{tabular}

* Mean of two determinations.

Subjects were supine 11 p.m. to 8 a.m., upright 8 a.m. to 11 p.m. 
TABLE II

Diurnal Plasma Cortisol, Corticosterone, Aldosterone, Renin Activity and Serum Potassium in Normal Subjects on a

\begin{tabular}{|c|c|c|c|c|c|c|c|c|c|c|}
\hline \multirow{2}{*}{$\begin{array}{l}\text { Sub- } \\
\text { ject }\end{array}$} & \multicolumn{5}{|c|}{ Cortisol } & \multicolumn{5}{|c|}{ Corticosterone } \\
\hline & $8 a^{*}$ & $11 \mathrm{a}$ & $5 p$ & $11 p$ & $8 a$ & $8 a^{*}$ & $11 a$ & $5 p$ & $11 p$ & $8 \mathbf{a}$ \\
\hline & \multicolumn{5}{|c|}{$\mu g / 100 \mathrm{ml}$} & \multicolumn{5}{|c|}{$\mu g / 100 \mathrm{ml}$} \\
\hline 1 & 11.7 & 8.7 & 10.6 & 12.8 & 15.6 & 0.38 & 0.31 & 0.24 & 1.36 & 0.94 \\
\hline 2 & 8.2 & - & 7.3 & 5.2 & 8.5 & 0.64 & - & 0.81 & 0.61 & 1.08 \\
\hline 3 & 14.1 & 9.0 & 12.2 & 4.8 & 11.2 & 0.85 & 0.40 & 0.98 & 0.50 & 1.10 \\
\hline 4 & 14.9 & 7.2 & 16.9 & 23.4 & 16.1 & 0.92 & 0.48 & 1.28 & - & 1.65 \\
\hline 5 & 12.6 & 11.3 & 11.8 & 10.8 & 19.6 & 0.49 & 0.26 & 0.14 & 0.45 & 0.72 \\
\hline Mean & 12.3 & 9.1 & 11.8 & 11.5 & 14.2 & 0.66 & 0.36 & 0.69 & 0.73 & 1.10 \\
\hline$\pm \mathrm{SEM}$ & \pm 1.1 & \pm 0.7 & \pm 1.4 & \pm 3.1 & \pm 1.8 & \pm 0.09 & \pm 0.02 & \pm 0.19 & \pm 0.18 & \pm 0.14 \\
\hline
\end{tabular}

* Mean of two determinations.

crease $(P<0.05)$ in the plasma aldosterone when the subject had been up for $3 \mathrm{hr}(84 \pm 6 \mathrm{ng} / 100 \mathrm{ml})$ (Table II). However, in contrast to the control day, there was no significant fall in the plasma aldosterone levels during the evening. The 11 p.m. value $(102 \pm 20 \mathrm{ng} / 100 \mathrm{ml})$ was significantly greater than both the 8 a.m. prediure- sis supine value $(55 \pm 7 \mathrm{ng} / 100 \mathrm{ml})$ and the 11 p.m. value on the control day $(33 \pm 5 \mathrm{ng} / 100 \mathrm{ml})$. The 8 a.m. postdiuresis supine value $(86 \pm 15 \mathrm{ng} / 100 \mathrm{ml})$ was also significantly greater $(P<0.05)$ than the 8 a.m. prediuresis supine value.

In contrast to the control day, both plasma cortisol and

TABLE III

Diurnal Plasma Cortisol, Corticosterone, Aldosterone, Renin Activity, Angiotensin II,

\begin{tabular}{|c|c|c|c|c|c|c|c|c|c|c|c|c|c|}
\hline \multirow{2}{*}{$\begin{array}{l}\text { Sub- } \\
\text { ject }\end{array}$} & \multirow{2}{*}{$\begin{array}{c}\text { Day } \\
\text { of } \\
\text { study }\end{array}$} & \multicolumn{4}{|c|}{ Cortisol } & \multicolumn{4}{|c|}{ Corticosterone } & \multicolumn{4}{|c|}{ Aldosterone } \\
\hline & & $8 a$ & $11 \mathrm{a}$ & $5 p$ & $11 p$ & $8 a$ & $11 \mathrm{a}$ & $5 p$ & $11 p$ & $8 a$ & $11 \mathrm{a}$ & 5p & $11 \mathrm{p}$ \\
\hline & & \multicolumn{4}{|c|}{$\mu \mathrm{g} / 100 \mathrm{ml}$} & \multicolumn{4}{|c|}{$\mu \mathrm{g} / 100 \mathrm{ml}$} & \multicolumn{4}{|c|}{$\mathrm{ng} / 100 \mathrm{ml}$} \\
\hline \multirow[t]{4}{*}{6} & 1 & 11 & 8 & 8 & 3 & 0.67 & 0.91 & 0.53 & 0.12 & 71 & 38 & 33 & 39 \\
\hline & 2 & 10 & 8 & 8 & 1 & 0.68 & 0.30 & 0.48 & 0.04 & 48 & 32 & 34 & 41 \\
\hline & 3 & 10 & 5 & 4 & - & 0.51 & 0.32 & 0.27 & 0.16 & 64 & 37 & 49 & 一 \\
\hline & 4 & 11 & & & & 0.89 & & & & 51 & & & \\
\hline \multirow[t]{4}{*}{7} & 1 & 12 & 10 & 6 & 1 & 1.38 & 0.24 & 0.29 & 0.15 & 86 & 61 & - & 36 \\
\hline & 2 & 10 & - & 8 & 1 & 1.04 & - & 0.69 & 0.11 & 164 & 160 & 82 & 65 \\
\hline & 3 & 12 & - & 3 & 1 & 0.79 & - & 0.09 & 0.05 & 89 & 62 & 56 & 68 \\
\hline & 4 & 15 & & & & - & & & & 115 & & & \\
\hline \multirow[t]{4}{*}{8} & 1 & 13 & 8 & 7 & 3 & 0.73 & 0.23 & 0.18 & 0.11 & 80 & 43 & 24 & 15 \\
\hline & 2 & 14 & - & 10 & - & 1.11 & - & 0.34 & - & 132 & - & 25 & - \\
\hline & 3 & 14 & 7 & 6 & - & 1.1 & 0.24 & 0.28 & - & - & 35 & 34 & 20 \\
\hline & 4 & 11 & & & & 0.63 & & & & 51 & & & \\
\hline \multirow[t]{4}{*}{9} & 1 & 10 & 6 & 3 & 1 & - & 0.27 & 0.18 & 0.06 & 101 & 43 & 21 & 21 \\
\hline & 2 & 10 & 6 & 7 & 3 & 0.39 & 0.04 & 0.04 & 0.02 & 61 & 47 & 25 & 24 \\
\hline & 3 & 14 & 8 & - & 8 & 0.32 & - & 0.13 & 0.10 & 65 & 38 & 54 & 19 \\
\hline & 4 & 11 & & & & 0.22 & & & & 65 & & & \\
\hline \multirow[t]{4}{*}{10} & 1 & 8 & 6 & 6 & 1 & 0.88 & 0.59 & 0.80 & 0.14 & 66 & 35 & 27 & 7 \\
\hline & 2 & 10 & 8 & 4 & 1 & 1.24 & 0.57 & 0.80 & 0.54 & 103 & 63 & 67 & 18 \\
\hline & 3 & 14 & 7 & 9 & 1 & - & 0.34 & 0.21 & 0.11 & 134 & 90 & 40 & - \\
\hline & 4 & 10 & & & & 0.52 & & & & 118 & & & \\
\hline Mean & & 11 & 7 & 6 & 2 & 0.77 & 0.37 & 0.35 & 0.14 & 88 & 56 & 41 & 31 \\
\hline \pm SEM & & \pm 1 & \pm 1 & \pm 1 & \pm 1 & \pm 0.08 & \pm 0.07 & \pm 0.06 & \pm 0.03 & \pm 7 & \pm 5 & \pm 5 & \pm 5 \\
\hline
\end{tabular}

* $10 \mathrm{mEq}$ sodium/100 mEq potassium diet. 
$10 \mathrm{mEq} \mathrm{Na/100} \mathrm{mEq} \mathrm{K} \mathrm{Diet} \mathrm{and} \mathrm{Normal} \mathrm{Activity} \mathrm{during} \mathrm{a} \mathrm{Day} \mathrm{When} \mathrm{They} \mathrm{Were} \mathrm{Diuresed} \mathrm{with} \mathrm{Furosemide}$

\begin{tabular}{|c|c|c|c|c|c|c|c|c|c|c|c|c|c|}
\hline \multicolumn{5}{|c|}{ Aldosterone } & \multicolumn{5}{|c|}{ Potassium } & \multicolumn{4}{|c|}{ Renin activity } \\
\hline $8 \mathrm{a}^{*}$ & $11 a$ & $5 p$ & $11 \mathrm{p}$ & $\mathbf{8 a}$ & $8 a^{*}$ & $11 \mathrm{a}$ & $5 p$ & $11 p$ & $8 a$ & $8 a^{*}$ & $11 \mathrm{a}$ & $5 p$ & $8 \mathbf{a}$ \\
\hline & \multicolumn{3}{|c|}{$n g / 100 \mathrm{ml}$} & \multicolumn{6}{|c|}{$m E_{q} /$ liter } & \multicolumn{4}{|c|}{$n g / 100 \mathrm{ml}$ per $3 \mathrm{hr}$} \\
\hline 70 & 69 & 91 & 133 & 131 & 4.5 & 4.5 & 3.9 & 3.9 & 3.9 & 784 & 1340 & 1780 & 1371 \\
\hline 33 & - & 45 & 37 & 32 & 4.5 & 4.4 & 3.9 & 3.9 & 3.7 & 1140 & 1280 & 1960 & 3800 \\
\hline 42 & - & 112 & 70 & 74 & 5.1 & 4.9 & 4.7 & 4.4 & 4.3 & 1210 & 1330 & 3260 & 2471 \\
\hline 80 & 93 & 63 & 162 & 110 & 4.7 & 4.6 & 5.0 & 4.6 & 3.8 & 975 & 1570 & 2410 & 2000 \\
\hline 46 & 190 & 66 & 109 & 83 & 4.6 & 4.0 & 3.8 & 3.1 & 4.7 & 325 & 730 & 1600 & 2000 \\
\hline 55 & 84 & 73 & 102 & 86 & 4.6 & 4.5 & 4.3 & 4.0 & 4.1 & 886 & 1250 & 2202 & 2328 \\
\hline \pm 7 & \pm 6 & \pm 10 & \pm 20 & \pm 15 & \pm 0.1 & \pm 0.1 & \pm 0.3 & \pm 0.3 & \pm 0.2 & \pm 121 & \pm 102 & \pm 268 & \pm 408 \\
\hline
\end{tabular}

corticosterone failed to show the same magnitude of fall during the day. The 11 p.m. cortisol level $(11.5 \pm 3.1 \mu \mathrm{g} /$ $100 \mathrm{ml}$ ) on the diuresis day was significantly greater than on the control day $(4.1 \pm 1.3 \mu \mathrm{g} / 100 \mathrm{ml})$. Although the 8 a.m. value postdiuresis $(14.2 \pm 1.8 \mu \mathrm{g} / 100 \mathrm{ml})$ was greater than the 8 a.m. value prediuresis $(12.3 \pm 1.1 \mu \mathrm{g} /$
$100 \mathrm{ml}$ ), the difference was not significant. Plasma corticosterone showed a similar altered pattern. However, the 8 a.m. level postdiuresis $(1.1 \pm 0.14 \mu \mathrm{g} / 100 \mathrm{ml})$ was significantly greater $(P<0.05)$ than prediuresis $(0.66 \pm$ $0.09 \mu \mathrm{g} / 100 \mathrm{ml}$ ).

The PRA pattern was also altered. There was a sig-

and Potassium in Normal Subjects Supine for 82 Consecutive $\mathrm{Hr}^{*}$

\begin{tabular}{|c|c|c|c|c|c|c|c|c|c|c|c|}
\hline \multicolumn{4}{|c|}{ Potassium } & \multicolumn{4}{|c|}{ Renin activity } & \multicolumn{4}{|c|}{ Angiotensin II } \\
\hline $8 \mathbf{a}$ & $11 \mathrm{a}$ & $5 p$ & $11 p$ & $8 \mathbf{a}$ & $11 \mathrm{a}$ & $5 p$ & $11 p$ & $8 a$ & $11 a$ & $5 p$ & $11 p$ \\
\hline \multicolumn{4}{|c|}{$m E q /$ liter } & \multicolumn{4}{|c|}{$\mathrm{ng} / 100 \mathrm{ml}$ per $3 \mathrm{hr}$} & \multicolumn{4}{|c|}{$p g / m l$} \\
\hline 3.8 & 3.9 & 3.4 & 一 & 600 & 910 & 730 & 630 & 40 & 65 & 37 & - \\
\hline 3.8 & 3.7 & - & 3.6 & 970 & 1040 & 650 & 840 & - & - & 一 & 一 \\
\hline 4.0 & - & 3.9 & 3.8 & 1120 & 1035 & 690 & 692 & 120 & 42 & - & - \\
\hline - & & & & 1200 & & & & - & & & \\
\hline - & 4.0 & 4.2 & 4.2 & 1200 & 1390 & 880 & 630 & 28 & - & 29 & - \\
\hline 4.0 & - & 4.2 & 3.7 & 1125 & 1300 & 890 & 670 & 49 & 31 & - & 37 \\
\hline- & - & 4.3 & 4.4 & 1140 & 1760 & 1136 & 945 & 43 & 一 & 34 & 45 \\
\hline 4.1 & & & & 1500 & & & & 70 & & & \\
\hline - & - & - & - & - & - & - & - & - & - & - & - \\
\hline - & - & - & - & - & - & - & - & - & - & - & - \\
\hline- & - & - & - & - & - & - & - & - & - & - & - \\
\hline- & & & & - & & & & - & & & \\
\hline 4.1 & 4.0 & 4.0 & 4.1 & 3992 & 2315 & 1120 & 1139 & 30 & 69 & 26 & 12 \\
\hline 4.3 & 4.0 & 4.1 & 3.8 & 2254 & 1833 & 964 & 1100 & 45 & 42 & 32 & 35 \\
\hline 4.2 & 3.9 & 4.0 & 4.2 & - & 1400 & 1270 & 640 & - & 28 & 14 & 31 \\
\hline 4.0 & & & & 1228 & & & & 25 & & & \\
\hline 4.2 & 4.2 & 4.1 & 3.8 & / 914 & 627 & 536 & 533 & 22 & $<10$ & - & 16 \\
\hline 3.8 & 4.2 & 4.0 & 3.8 & 1353 & 1271 & 467 & 294 & 125 & 25 & $<10$ & $<10$ \\
\hline 3.9 & 4.0 & 4.0 & 4.1 & 3462 & 867 & 583 & 367 & 24 & $<10$ & $<10$ & $<10$ \\
\hline 3.9 & & & & 1338 & & & & 47 & & & \\
\hline 4.0 & 4.0 & 4.0 & 4.0 & 1560 & 1312 & 826 & 707 & 51 & 36 & 24 & 24 \\
\hline \pm 0.1 & \pm 0.1 & \pm 0.1 & \pm 0.1 & \pm 240 & \pm 132 & \pm 72 & \pm 72 & \pm 9 & \pm 7 & \pm 4 & \pm 5 \\
\hline
\end{tabular}




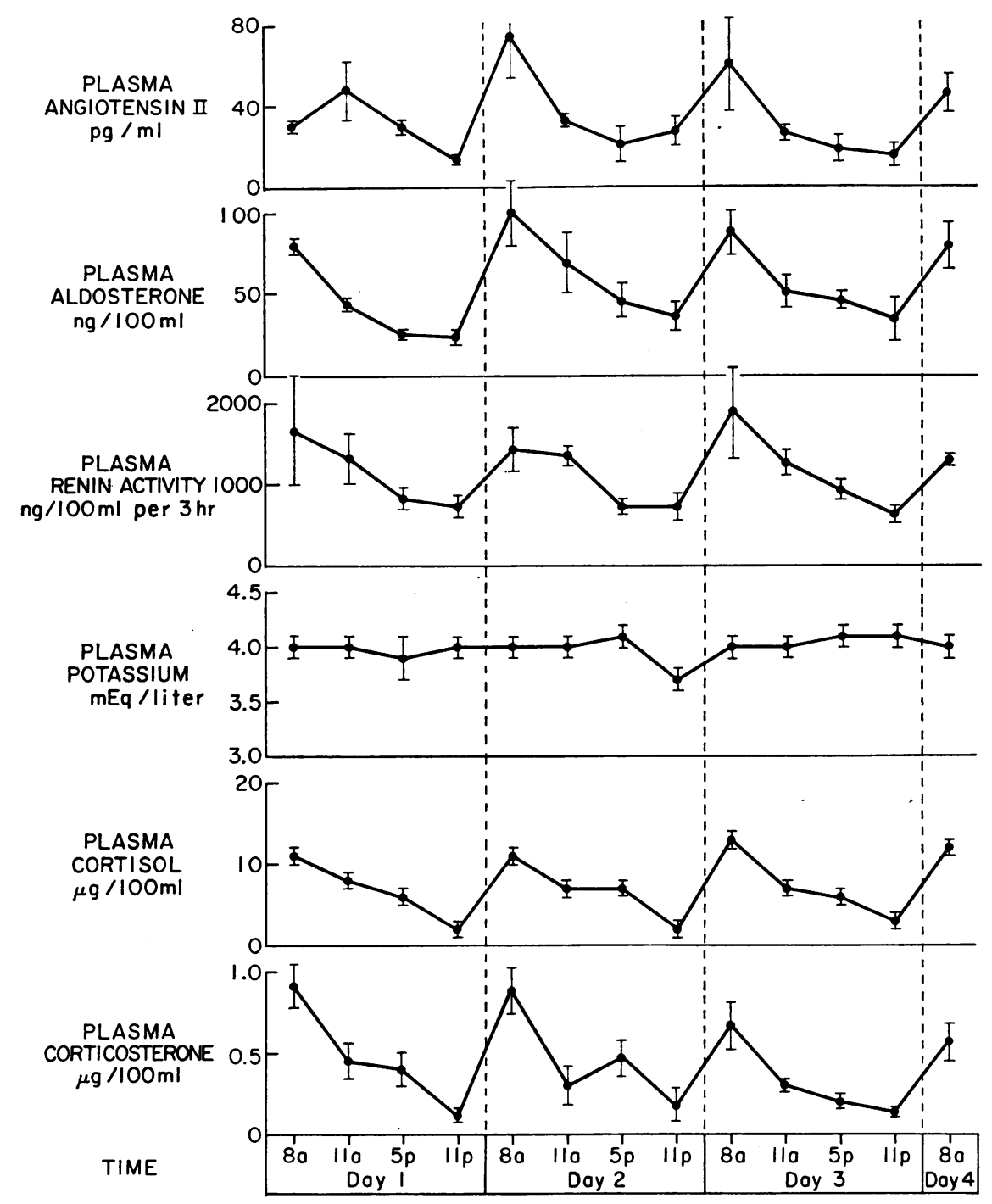

FIGURE 1 Diurnal variation in plasma angiotensin II, aldosterone, renin activity, potassium, cortisol, and corticosterone in supine normal subjects on a $10 \mathrm{mEq}$ sodium/100 $\mathrm{mEq}$ potassium diet.

nificant rise $(P<0.05)$ when the subjects went from supine to $3 \mathrm{hr}$ upright $(1250 \pm 102 \mathrm{ng} / 100 \mathrm{ml}$ per $3 \mathrm{hr})$, similar to the control day. However, the 5 p.m. level $(2202 \pm 268 \mathrm{ng} / 100 \mathrm{ml}$ per $3 \mathrm{hr}$ ) was significantly greater than both the 11 a.m. level and the 8 a.m. supine level. Furthermore, the 8 a.m. PRA postdiuresis (2328 \pm 408 $\mathrm{ng} / 100 \mathrm{ml}$ per $3 \mathrm{hr}$ ) was significantly greater than the 8 a.m. value prediuresis $(886 \pm 121 \mathrm{ng} / 100 \mathrm{ml}$ per $3 \mathrm{hr})$.

In contrast to the control day, there was a steady significant fall in the potassium levels from an 8 a.m. value of $4.6 \pm 0.1 \mathrm{mEq} /$ liter to an $11 \mathrm{p} . \mathrm{m}$ level of $4.0 \pm 0.3$ $\mathrm{mEq} /$ liter and a postdiuresis $8 \mathrm{a} . \mathrm{m}$. level of $4.1 \pm 0.2$
$\mathrm{mEq} /$ liter. The hematocrit and serum protein rose slightly but not significantly.

\section{Supine study-constant diet}

Chronic (low dietary sodium) volume depletion. The mean daily sodium excretion was $11 \pm 4,8 \pm 3$, and $5 \pm 1$ $\mathrm{mEq} /$ day on the 3 consecutive days of the study. The mean potassium excretion was $86 \pm 6,87 \pm 5$, and $84 \pm 4$ $\mathrm{mEq} /$ day.

The mean plasma aldosterone fell significantly during the course of the day (Table III, Fig. 1). The 8 a.m. level $(88 \pm 7 \mathrm{ng} / 100 \mathrm{ml})$ was significantly greater than the 11 a.m. value $(56 \pm 5 \mathrm{ng} / 100 \mathrm{ml})$ which was signifi- 


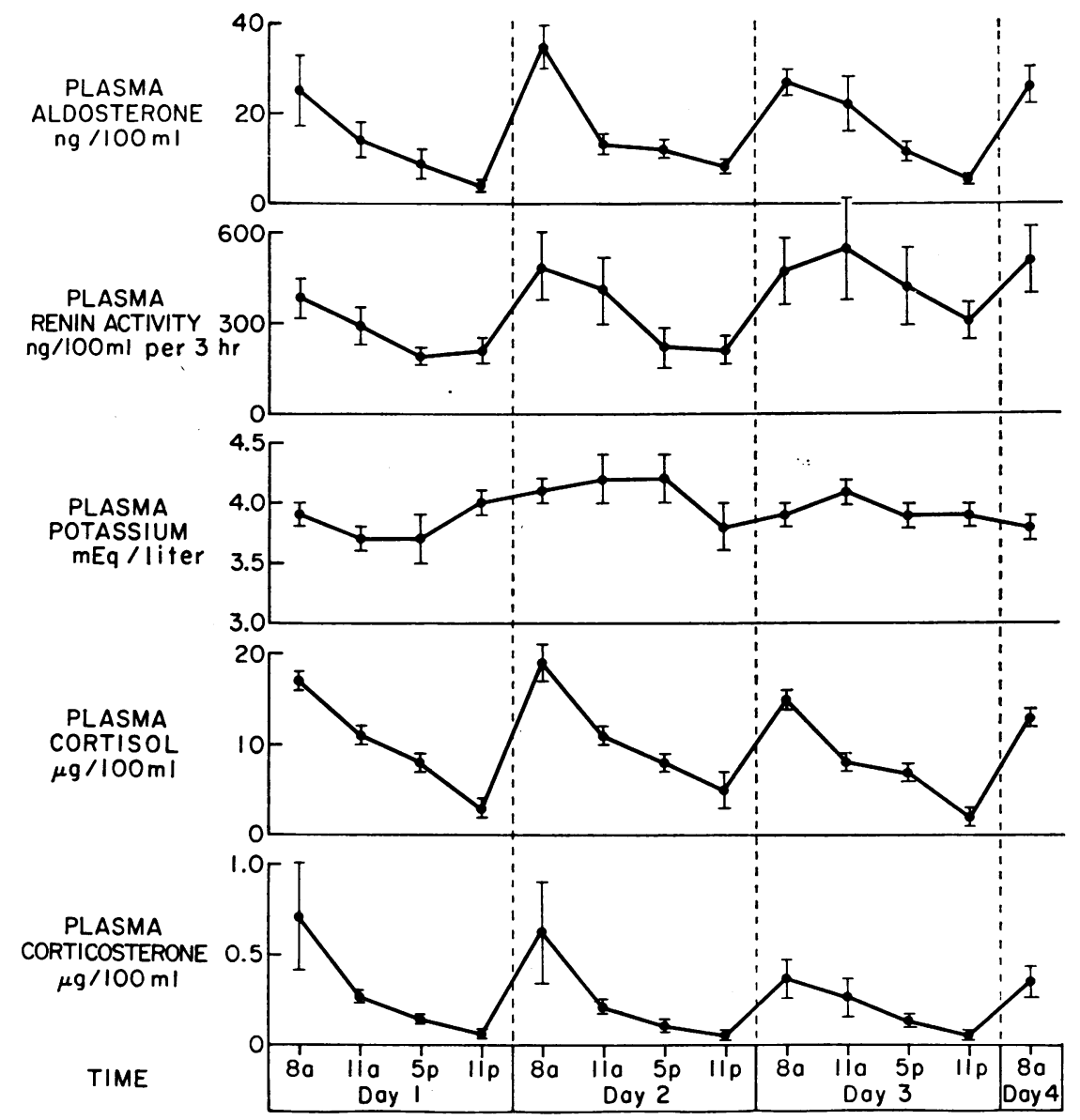

FIGURE 2 Diurnal variation in plasma aldosterone, renin activity, potassium, cortisol, and corticosterone in supine normal subjects on a $200 \mathrm{mEq}$ sodium/ $200 \mathrm{mEq}$ potassium diet.

cantly greater than the 11 p.m. value $(31 \pm 5 \mathrm{ng} / 100 \mathrm{ml})$. The 11 a.m. supine value was also significantly less than the 11 a.m. upright value $(100 \pm 17 \mathrm{ng} / 100 \mathrm{ml})$ from the previous protocol. Likewise, the 5 p.m. supine value $(41 \pm 5 \mathrm{ng} / 100 \mathrm{ml})$ was significantly less than the $5 \mathrm{p} . \mathrm{m}$. upright value $(68 \pm 4 \mathrm{ng} / 100 \mathrm{ml})$. However, there was no significant difference between the 11 p.m. supine and upright values. In some subjects, this circadian rhythm was more evident on the 2nd and 3rd days of this 3 day study. The 8 a.m. supine aldosterone was greater in this study than in the constant activity study $(55 \pm 7 \mathrm{ng} / 100 \mathrm{ml})$, probably because the hemorrhage induced 5-7 days before each study was $50 \%$ greater in the supine study.

Plasma cortisol showed the expected decline throughout the day with the $8 \mathrm{a} . \mathrm{m}$. value $(11 \pm 1 \mu \mathrm{g} / 100 \mathrm{ml})$ being significantly greater than the 11 p.m. value $(2 \pm 1$ $\mu \mathrm{g} / 100 \mathrm{ml})$. The mean plasma corticosterone at 8 a.m. $(0.77 \pm 0.08 \mu \mathrm{g} / 100 \mathrm{ml})$ was also significantly greater than the 11 p.m. value $(0.14 \pm 0.03 \mu \mathrm{g} / 100 \mathrm{ml})$. All three steroids, cortisol, corticosterone, and aldosterone, showed almost identical relative changes throughout the day and the correlation coefficient between them was highly significant $(P<0.001)$.

PRA showed a similar gradual fall throughout the day with the 8 a.m. value $(1560 \pm 240 \mathrm{ng} / 100 \mathrm{ml}$ per $3 \mathrm{hr})$ significantly greater than the 11 p.m. value $(707 \pm 72$ $\mathrm{ng} / 100 \mathrm{ml}$ per $3 \mathrm{hr}$ ). The 8 a.m. value was also significantly greater than the 5 p.m. value $(826 \pm 82 \mathrm{ng} / 100$ $\mathrm{ml}$ per $3 \mathrm{hr})$, and the $11 \mathrm{a} . \mathrm{m}$. value $(1312 \pm 132 \mathrm{ng} / 100$ $\mathrm{ml}$ per $3 \mathrm{hr}$ ) was significantly greater than both the 5 p.m. and the 11 p.m. levels (Table III, Fig. 1). The 8 a.m. plasma angiotensin II levels $(51 \pm 9 \mathrm{pg} / \mathrm{ml})$ were significantly greater $(P<0.05)$ than the 11 p.m. level $(25 \pm 5 \mathrm{pg} / \mathrm{ml})$. Thus, changes in PRA and angiotensin levels significantly correlated with changes in cortisol, corticosterone, and aldosterone. In contrast, the potas- 
TABLE IV

Diurnal Plasma Aldosterone, Cortisol, Corticosterone, Potassium, and Renin Activity

\begin{tabular}{|c|c|c|c|c|c|c|c|c|c|}
\hline \multirow{2}{*}{$\begin{array}{c}\text { Sub- } \\
\text { ject }\end{array}$} & \multirow{2}{*}{$\begin{array}{l}\text { Day } \\
\text { of } \\
\text { study }\end{array}$} & \multicolumn{4}{|c|}{ Cortisol } & \multicolumn{4}{|c|}{ Corticosterone } \\
\hline & & $8 a$ & $11 \mathrm{a}$ & $5 p$ & $11 \mathrm{p}$ & $8 \mathrm{a}$ & $11 \mathrm{a}$ & $5 p$ & $11 p$ \\
\hline & & \multicolumn{4}{|c|}{$\mu \mathrm{g} / 100 \mathrm{ml}$} & \multicolumn{4}{|c|}{$\mu \mathrm{g} / 100 \mathrm{ml}$} \\
\hline \multirow[t]{4}{*}{6} & 1 & 18 & 7 & 9 & 4 & 1.75 & - & 0.21 & 0.07 \\
\hline & 2 & - & 8 & - & - & 1.84 & 0.31 & 一 & - \\
\hline & 3 & 18 & 8 & 8 & 2 & 0.82 & 0.75 & 0.23 & 0.04 \\
\hline & 4 & 一 & & & & 0.60 & & & \\
\hline \multirow[t]{4}{*}{7} & 1 & 16 & 15 & 9 & 4 & 0.38 & 0.13 & 0.06 & 0.05 \\
\hline & 2 & 21 & 12 & 6 & 2 & 0.20 & 0.16 & 0.12 & 0.05 \\
\hline & 3 & 15 & 8 & 5 & 1 & 0.20 & 0.16 & 0.04 & 0.06 \\
\hline & 4 & 15 & & & & 0.26 & & & \\
\hline \multirow[t]{4}{*}{11} & 1 & 18 & 10 & 7 & 4 & 0.46 & 0.33 & 0.20 & 0.08 \\
\hline & 2 & 13 & 10 & 6 & 3 & 0.38 & 0.25 & 0.15 & 0.06 \\
\hline & 3 & 14 & 7 & 8 & 4 & 0.26 & 0.15 & 0.07 & 0.08 \\
\hline & 4 & 15 & & & & 0.46 & & & \\
\hline \multirow[t]{4}{*}{12} & 1 & 17 & 16 & 8 & 2 & 0.24 & 0.28 & 0.16 & 0.07 \\
\hline & 2 & 17 & 14 & 11 & 8 & 0.22 & 0.13 & 0.07 & 0.05 \\
\hline & 3 & - & 9 & 5 & 2 & 0.13 & 0.12 & 0.13 & 0.04 \\
\hline & 4 & - & & & & 0.10 & & & \\
\hline \multirow[t]{4}{*}{13} & 1 & - & 9 & 5 & 2 & - & 0.36 & 0.11 & 0.04 \\
\hline & 2 & 23 & 12 & 7 & 6 & 0.53 & 0.23 & 0.08 & 0.04 \\
\hline & 3 & 14 & 7 & 7 & 2 & 0.47 & 0.17 & 0.22 & 0.06 \\
\hline & 4 & 10 & & & & 0.36 & & & \\
\hline Mean & & 16 & 10 & 7 & 3 & 0.51 & 0.25 & 0.13 & 0.05 \\
\hline \pm SEM & & \pm 1 & \pm 1 & \pm 1 & \pm 1 & \pm 0.11 & \pm 0.04 & \pm 0.02 & \pm 0.01 \\
\hline
\end{tabular}

sium levels showed no diurnal changes throughout the day.

Volume expansion study. The daily mean sodium excretion on the 3 days of this study was $245 \pm 31,209 \pm 11$, and $185 \pm 23 \mathrm{mEq} /$ day. Although there was a tendency for the sodium excretion to be greater on the 1st supine day, this was not significant. Mean daily potassium excretion was $171 \pm 7,157 \pm 10$, and $154 \pm 13 \mathrm{mEq} /$ day on each one of the days.

Mean plasma aldosterone concentration declined throughout the day. The mean 8 a.m. level $(28 \pm 3 \mathrm{ng} / 100$ $\mathrm{ml})$ was significantly greater than the 11 a.m. level $(16 \pm 3 \mathrm{ng} / 100 \mathrm{ml})$, the $5 \mathrm{p} . \mathrm{m}$. level $(11 \pm 2 \mathrm{ng} / 100 \mathrm{ml})$, and the 11 p.m. level $(6 \pm 1 \mathrm{ng} / 100 \mathrm{ml})$. The plasma corticosterone and cortisol mean levels showed a similar fall during the day; the mean levels at 8 a.m. were significantly greater than at 11 p.m. (Table IV, Fig. 2).

As in the low sodium-supine study, PRA showed a constant decline parallel to that of cortisol, corticosterone, and aldosterone. The mean 8 a.m. PRA value (471士 $80 \mathrm{ng} / 100 \mathrm{ml}$ per $3 \mathrm{hr}$ ) was significantly greater than the 11 p.m. value $(240 \pm 28 \mathrm{ng} / 100 \mathrm{ml}$ per $3 \mathrm{hr}$ ) (Table IV, Fig. 2). Potassium levels similarly showed no significant changes during the course of the study and were not significantly correlated with either PRA, cortisol, corticosterone, or aldosterone.
In comparing the plasma steroid levels on the high and low sodium diets, cortisol showed no significant differences and aldosterone was always significantly higher on the low sodium intake at all time periods. In contrast, while corticosterone levels were not significantly different at 8 and 11 a.m., they were significantly greater on the low sodium diet at 5 and 11 p.m.

\section{DISCUSSION}

In the present study, upright posture, diuresis, or alterations in sodium balance were associated with parallel changes in PRA and aldosterone. Under some circumstances, changes in plasma aldosterone also coincided with changes in plasma cortisol, corticosterone, or potassium. However, under all circumstances, changes in plasma aldosterone were associated with parallel changes in PRA and/or angiotensin II levels. These findings support the concept that angiotensin II is the dominant factor in the control of aldosterone with volume manipulation in normal man. Some investigators have not always found such a close association (11-13, 24). In some instances, the experimental design or methodologic differences might explain this. The present study was designed to carefully control other factors which could alter the levels of renin activity or aldosterone and 
in Normal Subjects on a $200 \mathrm{mEq}$ Sodium/200 mEq Potassium Diet Supine for $82 \mathrm{Hr}$

\begin{tabular}{|c|c|c|c|c|c|c|c|c|c|c|c|}
\hline \multicolumn{4}{|c|}{ Aldosterone } & \multicolumn{4}{|c|}{ Potassium } & \multicolumn{4}{|c|}{ Renin activity } \\
\hline $8 \mathbf{a}$ & $11 \mathrm{a}$ & $5 p$ & $11 p$ & $8 \mathbf{a}$ & $11 \mathrm{a}$ & $5 p$ & $11 p$ & $8 a$ & $11 \mathrm{a}$ & $5 p$ & $11 \mathrm{p}$ \\
\hline \multicolumn{4}{|c|}{$\mathrm{ng} / 100 \mathrm{ml}$} & \multicolumn{4}{|c|}{$m E q /$ liter } & \multicolumn{4}{|c|}{$n g / 100 \mathrm{ml}$ per $3 \mathrm{hr}$} \\
\hline 11 & 8 & 4 & 4 & 3.8 & 3.9 & 3.2 & - & 260 & 220 & 110 & 210 \\
\hline - & 11 & - & - & - & 3.5 & - & - & 770 & 450 & 470 & 270 \\
\hline 16 & 14 & 14 & 5 & 3.4 & 3.9 & 3.6 & 3.8 & 556 & 883 & 640 & 420 \\
\hline 19 & & & & 3.6 & & & & 760 & & & \\
\hline 10 & 3 & 4 & 1 & 3.9 & 3.9 & 3.8 & 3.9 & 360 & 170 & 150 & 63 \\
\hline 21 & 12 & 7 & 7 & 4.0 & 3.8 & 3.9 & 3.8 & 250 & 150 & 100 & 160 \\
\hline 24 & 13 & 4 & 1 & 4.0 & 4.1 & 3.9 & 3.7 & 240 & 260 & 170 & 94 \\
\hline 26 & & & & 3.6 & & & & 270 & & & \\
\hline 48 & 15 & 7 & 6 & 3.5 & 3.6 & 3.7 & 3.8 & 650 & 488 & 192 & 223 \\
\hline 46 & 10 & 7 & 7 & 3.7 & 4.5 & 3.6 & 3.2 & 486 & 385 & 142 & 194 \\
\hline 39 & 16 & 16 & 7 & 3.8 & 3.8 & 3.8 & - & 357 & 80 & 194 & 253 \\
\hline 40 & & & & 3.9 & & & & 760 & & & \\
\hline 29 & 29 & 22 & 5 & 4.0 & 3.9 & 4.3 & 4.2 & 450 & 460 & 310 & 350 \\
\hline 39 & 21 & 15 & 13 & 4.5 & 4.6 & 4.5 & 4.5 & 790 & 850 & 330 & 280 \\
\hline 30 & 44 & 16 & 5 & 4.3 & 4.1 & 4.3 & 4.1 & 920 & 1140 & 860 & 480 \\
\hline 15 & & & & 4.2 & & & & 500 & & & \\
\hline- & 17 & 7 & 6 & 4.3 & 3.1 & 3.7 & 4.1 & 260 & 140 & 200 & 180 \\
\hline 32 & 9 & 18 & 6 & 4.3 & 4.4 & 4.7 & 3.7 & 180 & 220 & 83 & 150 \\
\hline 24 & 24 & 7 & 9 & 4.1 & 4.6 & 3.9 & 4.1 & 371 & 400 & 267 & 277 \\
\hline 28 & & & & 3.8 & & & & 220 & & & . \\
\hline 28 & 16 & 11 & 6 & 3.9 & 4.0 & 3.9 & 3.9 & 471 & 420 & 281 & 240 \\
\hline \pm 3 & \pm 3 & \pm 2 & \pm 1 & \pm 0.1 & \pm 0.1 & \pm 0.1 & \pm 0.1 & \pm 50 & \pm 78 & \pm 56 & \pm 28 \\
\hline
\end{tabular}

thereby artifactually produce dissociation between them. Dietary intake of sodium and potassium as well as the timing of sampling to eating were rigidly regulated since it has been shown that changes in either one can produce significant changes in the plasma levels of aldosterone and/or PRA $(4,6,25)$. Because hemorrhage has been shown to induce changes in renin activity and aldosterone secretion (26), an equal volume of the subject's blood was reinfused during the course of the study as samples were obtained. Activity of the patients before and during the time of sampling was also standardized. In studies of over 50 normal subjects on supine protocols, we have observed that as many as $25 \%$ excrete a significantly greater amount of sodium on the 1st day supine than on previous days with normal activity or subsequent supine days. Because this increased natriuresis might alter the normal pattern of aldosterone in some subjects, two additional days of sampling were performed.

The absolute plasma aldosterone values on both high and low sodium intake are in reasonable agreement with other investigators (Table V). The higher plasma level on a high sodium diet in the present study is probably the result of the greater daily potassium intake $(200 \mathrm{mEq})$ and to a lesser extent the hemorrhage induced 5-8 days before sampling. The slightly higher mean value in the present study on a $10 \mathrm{mEq}$ sodium/ $100 \mathrm{mEq}$ potassium intake could reflect the greater potassium intake, the lower sodium intake and/or the previous hemorrhage. The mean PRA on the low sodium study of Michelakis and Horton (13) was only $25 \%$ of the value obtained in the present study and in the study of Gordon, Wolfe, Island, and Liddle (29). There is no apparent explanation for this difference, particularly since PRA in Michelakis' and Horton's study, like the angiotensin II levels in the study of Best, Coghlan, Bett, Cran, and Scoggins (24), did not significantly change when sodium intake was restricted.

It has been well established that there is a diurnal pattern to the excretion of aldosterone with a greater excretion in the daytime than at night $(14,30,31)$. Whether this pattern was anything more than a reflection of the diurnal posture of the subjects was unclear since most investigators had found no consistent change in excretion of aldosterone when posture was eliminated $(32,33)$. Analysis of plasma values of aldosterone have produced conflicting results. An early study (34) showed no significant variation in the level of aldosterone (obtained every $6 \mathrm{hr}$ ) in recumbent subjects. More recently, Michelakis and Horton (13) demonstrated that there is a diurnal variation of plasma aldosterone independent of posture in normal subjects. 
TABLE V

Comparison of Plasma Aldosterone and Renin Activity Measured in Several Studies

\begin{tabular}{|c|c|c|c|c|c|}
\hline \multirow[b]{2}{*}{ Investigator } & \multicolumn{2}{|c|}{ Urine excretion } & \multirow{2}{*}{$\begin{array}{c}\text { Plasma } \\
\text { aldosterone }\end{array}$} & \multirow[b]{2}{*}{ *Method } & \multirow{2}{*}{$\begin{array}{l}\text { Renin } \\
\text { activity }\end{array}$} \\
\hline & Sodium & Potassium & & & \\
\hline & \multicolumn{2}{|c|}{$m E q / d a y$} & $n g / 100 \mathrm{ml}$ & & $\begin{array}{c}n g / 100 \mathrm{~m} \\
\text { per } 3 \mathrm{hr}\end{array}$ \\
\hline \multirow{2}{*}{ Michelakis and Horton (13) } & 150 & 82 & 9.8 & DID & 197 \\
\hline & $10-20$ & 82 & 24.1 & DID & 274 \\
\hline Balikian et al. (27) & 128 & 50 & 2.15 & DID & - \\
\hline \multirow[t]{2}{*}{ Ford et al. (28) } & 146 & $65-73$ & 9.1 & Calc. & - \\
\hline & 16 & $65-73$ & 45.5 & Calc. & - \\
\hline Gordon et al. (29) & 10 & Unspecified & - & & 800 \\
\hline \multirow[t]{2}{*}{ Present study } & 161 & 213 & 28 & RIA & 471 \\
\hline & 6 & 94 & 55 & RIA & 866 \\
\hline
\end{tabular}

* DID, double-isotope derivative; Calc., calculated from secretion rate and metabolic clearance rate; RIA, radioimmunoassay.

However, the timing of the circadian rhythm was dependent upon dietary intake of sodium. With intake of normal amounts of sodium, the peak plasma aldosterone value occurred at midnight, while on a low sodium intake, it occurred between 6 a.m. and noon (similar with that of cortisol). PRA also showed a circadian rhythm, but peak values occurred at 12 midnight on both diets. Thus, there was an unexplained dissociation between PRA and plasma aldosterone on a low sodium intake. The present study confirms that there is a diurnal variation of plasma aldosterone independent of the upright posture. However, in contrast to what had previously been reported (13), there was no alteration in the diurnal pattern of plasma aldosterone when dietary sodium intake was changed from $10 \mathrm{mEq}$ to $200 \mathrm{mEq} /$ day. Thus, no dissociation between PRA and aldosterone was seen in the present study. The PRA pattern is similar to what Gordon et al. reported in sodium restricted supine subjects with a peak value between 4 and 8 a.m. (29). The difference between the present study and the previous ones may reflect methodologic differences. In Michelakis' and Horton's study (13), a single day's sampling was obtained to determine the pattern of plasma aldosterone and PRA. As has been indicated earlier in some of our subjects, the plasma aldosterone cycle obtained during the 1st day supine was sometimes different than during the subsequent days. Secondly, they did not replace the blood lost during the sampling period. Since the last sample drawn in their study was at midnight, the increased levels found at that time for both PRA and aldosterone may in part be a reflection of the hemorrhage induced.

Gordon et al. (29) have also measured PRA throughout the day in subjects maintaining normal upright posture. In spite of this constant stimulus for renin release, the circadian rhythm of PRA in their study and the plasma aldosterone in the present one were maintained with a low value occurring in the late evening. The mechanism by which the circadian pattern in plasma aldosterone is produced and how it is maintained even in the face of upright posture is unclear from the present or previous studies. It has been amply demonstrated that ACTH can stimulate both excretion, secretion, and plasma levels of aldosterone $(1,17,35)$. Since in the supine studies, the plasma levels of aldosterone, cortisol, and corticosterone all changed in parallel fashion, they may all be related to the same stimulus, i.e., the circadian variation in ACTH release. Alternatively, because PRA varied in a fashion parallel to aldosterone, it may be the direct stimulus. It has been shown in the rat (36), but not in man (37), that ACTH can alter renin release. Since PRA varied in a manner parallel to plasma aldosterone, ACTH may be affecting both renin release and adrenal glucocorticoid secretion or another, presumably central nervous system factor, may be producing a synchronous release of both ACTH and renin.

There are circadian changes in the levels of a variety of substances in the blood or urine of normal man (38). Therefore, the parallel changes in PRA and aldosterone in supine subjects do not necessarily mean that aldosterone follows renin activity, but may simply reflect part of a general diurnal cycling. Acute alterations in the cycle were therefore produced to determine if plasma aldosterone would still follow alterations in PRA. Assuming the upright posture produced a significant 150 $200 \%$ parallel increase in both PRA and plasma aldosterone without any alteration in plasma corticosterone and cortisol. These changes are in agreement with those of Michelakis and Horton (13) for subjects on a low sodium intake and with Balikian, Brodie, Dale, 
Melby, and Tait (27) for subjects on a high sodium intake.

The second alteration of the normal circadian pattern was produced by acute volume depletion. Previous studies have shown that when a $2-3 \%$ decrease in body weight is produced by acute diuresis, there is a two to fivefold increase in PRA and aldosterone secretion in normal subjects $(17,39)$. Rosenthal, Boucher, Nowaczynski, and Genest (16) have shown that acute administration of furosemide produced a three to fourfold increase in PRA after $60 \mathrm{~min}$ and a doubling of plasma aldosterone after $2 \mathrm{hr}$. Results of the present study show a similar parallel increase in renin activity and aldosterone in response to acute diuresis. Furthermore, the acute diuresis completely abolished the usual evening fall in PRA and aldosterone. Although cortisol and corticosterone diurnal patterns were also altered by the diuresis, the changes were quantitatively less significant than those observed for PRA and aldosterone.

At times in the present study, changes in plasma aldosterone paralleled presumed changes in ACTH secretion as reflected in changes in plasma levels of corticosterone and cortisol. In the supine study, there was a strong correlation between plasma levels of corticosterone, cortisol, and aldosterone. However, in the constant activity study where the normal circadian release of PRA was altered, plasma aldosterone but not cortisol and corticosterone followed the changes in PRA. Changes in aldosterone could also be dissociated from changes in potassium, another aldosterone stimulus. Serum potassium levels during the supine study did not change when there was a significant fall in both plasma aldosterone and PRA. Likewise, in the volume depletion study, there was a negative correlation with the serum potassium falling during the day, while the plasma aldosterone rose.

It is clear from the present study that changes in volume, dietary sodium, or posture produce parallel changes in plasma aldosterone, plasma angiotensin II, and PRA. While other factors may modify the response and presumably the synchrony between PRA and aldosterone secretion under the assigned criteria of the present study, there was a very close correlation between renin activity, angiotensin II, and aldosterone in young, healthy individuals. The data suggest that plasma aldosterone is under the dominant control of renin activity and angiotensin II in these circumstances. This is critically important since in some diseases $(15,17$, 40-43) a dissociation between PRA and aldosterone secretion has been reported with sodium manipulation and/or volume depletion. The results of the present study would imply that in these diseases other factors are influencing the levels of PRA and aldosterone.

\section{ACKNOWLEDGMENTS}

These investigations were supported in part by the John A. Hartford Foundation, grant 9893 , and in part by Public Health Service Training grant AM-5100-14 from the National Institute of Arthritis and Metabolic Diseases. The clinical studies were carried out in The Clinical Research Center of the Peter Bent Brigham Hospital, supported by grant 8-M01-FR-31-06.

\section{REFERENCES}

1. Liddle, G. W., L. E. Duncan, Jr., and F. C. Bartter. 1956. Dual mechanism regulating adrenocortical function in man. Amer. J. Med. $21: 380$.

2. Laragh, J. H., and H. C. Stoerk. 1957. A study of the mechanism of secretion of the sodium retaining hormone (aldosterone). J. Clin. Invest. 36: 383.

3. Binnion, P. F., J. O. Davis, T. C. Brown, and M. J. Olichney. 1965. Mechanisms regulating aldosterone secretion during sodium depletion. Amer. J. Physiol. 208: 655.

4. Gross, F. 1968. The regulation of aldosterone secretion by the renin-angiotensin system under various conditions. Acta Endocrinol. Suppl. 124: 41.

5. Laragh, J. H., and H. C. Stoerk. 1955. On the mechanism of secretion of the sodium-retaining hormone (aldosterone). J. Clin. Invest. 34 : 913.

6. Dluhy, R. G., R. H. Underwood, and G. H. Williams. 1970. Influence of dietary potassium on plasma renin activity in normal man. J. Appl. Physiol. 28: 299.

7. Ganong, W. F., E. G. Bigleri, and P. J. Mulrow. 1966. Mechanisms regulating adrenocortical secretion of aldosterone and glucocorticoids. Recent Prog. Horm. Res. 22: 381 .

8. Williams, G. H., R. G. Dluhy, and R. H. Underwood. 1970. The relationship of dietary potassium intake to the aldosterone stimulating properties of ACTH. Clin. Sci. (London). 39 : 489.

9. Venning, E. H., I. Dyrenfurth, J. B. Dossetor, and J. C. Beck. 1962. Influence of alterations in sodium intake on urinary aldosterone response to corticotropin in normal individuals and patients with essential hypertension. Metab. (Clin. Exp.). 11: 254.

10. Müller, J., and W. H. Ziegler. 1968. Stimulation of aldosterone biosynthesis in vitro by serotonin. Acta Endocrinol. 59: 23.

11. Müller, J. 1971. Regulation of Aldosterone Biosynthesis. Springer-Verlag New York Inc., New York. 108.

12. Blair-West, J. R., M. Cain, K. Catt, J. P. Coghlan, D. A. Denton, J. W. Funder, B. A. Scoggins, and R. D. Wright. 1969. Aldosterone: regulation and biosynthesis. In Progress in Endocrinology. C. Gual, editor. Excerpta Medica Foundation Publishers, Amsterdam. 276.

13. Michelakis, A. M., and R. Horton. 1970. The relationship between plasma renin and aldosterone in normal man. Circ. Res. Suppl. 26-27: I-185.

14. Luetscher, J. A., Jr., and A. H. Lieberman. 1958. Aldosterone. Arch. Intern. Med. $102: 314$.

15. Luetscher, J. A., M. H. Weinberger, A. J. Dowdy, G. W. Nokes, H. Balikian, A. Brodie, and S. Willoughby. 1969. Effect of sodium loading, sodium deprivation and posture on plasma aldosterone concentration and renin activity in hypertensive patients. J. Clin. Endocrinol. Metab. 29: 1310.

16. Rosenthal, J., R. Boucher, W. Nowaczynski, and J. Genest. 1968. Acute changes in plasma volume, renin 
activity, and free aldosterone levels in healthy subjects following furosemide administration. Can. J. Physiol. Pharmacol. 46: 85.

17. Williams, G. H., L. I. Rose, R. G. Dluhy, D. McCaughn, P. I. Jagger, R. B. Hickler, and D. P. Lauler. 1970. Abnormal responsiveness of the renin aldosterone system to acute stimulation in patients with essential hypertension. Ann. Intern. Med. 72: 317.

18. Kliman, B., and R. E. Peterson. 1960. Double isotope derivative assay of aldosterone in biologic extracts. J. Biol. Chem. 235 : 1639.

19. Brodie, A. H., N. Shimizu, S. A. S. Tait, and J. F. Tait. 1967. A method for the measurement of aldosterone in peripheral plasma using ${ }^{3} \mathrm{H}$-acetic anhydride. J. Clin. Endocrinol. Metab. 27: 997.

20. Underwood, R. H., and G. H. Williams. 1972. Simultaneous determination of aldosterone, corticosterone and cortisol in peripheral plasma by displacement analysis techniques. J. Lab. Clin. Med. 79: 848.

21. Nugent, C. A., and D. M. Mayes. 1966. Plasma corticosteroids determined by use of corticosteroids binding globulin and dextran-coated charcoal. J. Clin. Endocrinol. Metab. 26: 1116.

22. Emanuel, R., J. P. Cain, and G. H. Williams. 1972. Simultaneous determination of angiotensin $\mathrm{I}$ and angiotensin II in unextracted plasma by double antibody radioimmunoassay. J. Lab. Clin. Med. In press.

23. Snedecor, G. W., and W. G. Cochran. 1967. Statistical Methods. Iowa State University Press, Ames, Iowa. 6th edition. 91, 172.

24. Best, J. B., J. P. Coghlan, J. H. N. Bett, E. J. Cran, and B. A. Scoggins. 1971. Circulating angiotensin II and aldosterone levels during dietary sodium restriction. Lancet. 2: 1353.

25. Dluhy, R. G., L. Axelrod, R. H. Underwood, and G. H. Williams. 1972. Studies of the control of plasma adlosterone concentration in normal man. II. Effect of dietary potassium and acute potassium infusion. J. Clin. Invest. In press.

26. Skillman, J. J., D. P. Lauler, R. B. Hickler, J. H. Lyons, J. E. Olsen, M. R. Ball, and F. D. Moore. 1967. Hemorrhage in normal man. Ann. Surg. 166: 865.

27. Balikian, H. M., A. H. Brodie, S. L. Dale, J. C. Melby, and J. F. Tait. 1968. Effect of posture on the metabolic clearance rate, plasma concentration and blood production rate of aldosterone in man. J. Clin. Endocrinol. Metab. 28: 1630 .

28. Ford, H. C., H. P. Pieters, and R. E. Bailey. 1968. Aldosterone and sodium conservation, metabolic clearance and secretion-excretion rates of aldosterone in normal subjects. J. Clin. Endocrinol. Metab. 28 : 451.

29. Gordon, R. D., L. K. Wolfe, D. P. Island, and G. W. Liddle. 1966. A diurnal rhythm in plasma renin activity in man. J. Clin. Invest. 45: 1587.
30. Bartter, F. C., C. S. Delea, and F. Halberg. 1962. A map of blood and urinary changes related to circadian variations in adrenal cortical function in normal man. Ann. N. Y. Acad. Sci. 98: 969.

31. Venning, E. H., I. Dyrenfurth, and C. J. P. Giroud. 1956. Aldosterone excretion in healthy persons. J. Clin. Endocrinol. Metab. 16: 1326.

32. Muller, A. F., E. L. Manning, and A. M. Riondel. 1958. Influence of position and activity on secretion of aldosterone. Lancet. 1: 711 .

33. Wolfe, L. K., R. D. Gordon, D. P. Island, and G. W. Liddle. 1966. An analysis of factors determining the circadian pattern of aldosterone excretion. J. Clin. Endocrinol. Metab. 26: 1261.

34. Wolff, H. P., and M. Torbrica. 1963. Determination of plasma aldosterone. Lancet. 1: 1346.

35. Horton, R. 1969. Stimulation and suppression of aldosterone in plasma in normal man and primary aldosteronism. J. Clin. Invest. 48: 1230.

36. Hanger-Klevene, J. H., H. Brown, and N. Fleischer. 1969. ACTH stimulation and glucocorticoid inhibition of renin release in the rat. Proc. Soc. Exp. Biol. Med. $131: 539$.

37. Newton, M. A., and J. H. Laragh. 1968. Effect of corticotropin on aldosterone excretion and plasma renin in normal subjects, in essential hypertension, and in primary aldosteronism. J. Clin. Endocrinol. Metab. 28: 1006.

38. Mills, J. N. 1966. Human circadian rhythms. Physiol. Rev. 46: 128.

39. Espiner, E. A., J. R. Tucci, P. I. Jagger, and D. P. Lauler. 1967. The effect of acute diuretic induced volume depletion on aldosterone secretion in normal man. Clin. Sci. (London). 33: 125.

40. Collins, R. D., M. R. Weinberger, A. J. Dowdy, D. W. Nokes, C. M. Gonzales, and J. A. Luetscher 1970. Abnormally sustained aldosterone secretion during salt loading of patients with various forms of benign hypertension; relationship to plasma renin activity. $J$. Clin. Invest. $49: 1415$.

41. Christlieb, A. R., R. B. Hickler, D. P. Lauler, and G. H. Williams. 1969. Hypertension with inappropriate aldosterone stimulation: a syndrome. N. Engl. J. Med. 281: 128

42. Chin, R. H., J. A. Brown, R. Fraser, S. M. Heron, A. F. Lever, L. Murchison, and J. I. S. Robertson. 1970. The natriuresis of fasting: relationship to changes in plasma renin and plasma aldosterone concentration. Clin. Sci. (London). 39: 437.

43. Mitra, S., S. M. Genuth, L. B. Berman, and V. Vertes. 1972. Aldosterone Secretion in anephric patients. $N$. Engl. J. Med. 286: 61. 